\title{
Dynamic Parameters Tuning Using Structural Dynamic Modification
}

\author{
Miloš Musil ${ }^{1}$, René Havelka ${ }^{2, *}$, Ondrej Chlebo ${ }^{3}$, Ferdinand Havelka ${ }^{4}$ and Branislav Hučko ${ }^{5}$ \\ ${ }^{1}$ Faculty of Mechanical Engineering, Slovak University of Technology, Námestie slobody 17, 81231 Bratislava 1, Slovakia
}

\begin{abstract}
Significant vibration of construction components are unwanted and dangerous problems in the modern engineering. Nevertheless, undesirable levels of vibration can occur in already existing constructions. Therefore, additional modal and spectral parameter modification, to reach the desired vibration reducing, is required. This work presents structural dynamic modification methodology that allows the modal properties of a structure to be effectively changed. As the demonstration of this method, the example of a cantilever beam structure modified by aluminium foam layer elements was chosen. This example represents inertial, stiffness and damping modification aimed to tuning of modal and spectral parameters of the original structure.
\end{abstract}

Keywords: dynamic parameters tuning; structural dynamic modification; aluminium foam.

\section{Introduction}

Dynamic parameters tuning is a required operation when significant vibration of a construction component is expected. Dynamic parameters tuning using structural dynamic modification represents modal-spectral parameters changes by adding substructures to the original to avoid resonances and reduce the level of vibration. Those substructures, which influent mass, stiffness and damping matrices of the original system, can increase or decrease natural frequencies and influents corresponding mode shapes according to the desire behaviour of the structure. Additional tuning of dynamic parameters performed by structural dynamic modification is a useful operation to adapt the structure to changed operation conditions. Modal and spectral parameters of the modified structure can be determined by analytical approach using structural coefficient matrices of modifying substructures and modal parameters of the original structure, that can be obtained experimentally.

\section{Structural modification}

This chapter is aimed to the modal synthesis explanation of the original structure and substructures to determine modal parameters of the resulting system.

The original structure is represented by the proportionally damped system whose eigenvalue solution has the following form.

$$
\left(\boldsymbol{K}_{0}-\boldsymbol{M}_{0} \omega_{0 j}^{2}\right) \boldsymbol{v}_{0 j}=\mathbf{0}
$$

Where stiffness and mass parameters are represented by coefficient matrices $\boldsymbol{K}_{\mathbf{0}}$ and $\boldsymbol{M}_{0}$ and $j^{\text {th }}$ eigen mode and natural frequency for this mode by symbols $\boldsymbol{v}_{0 j}$ and $\omega_{0 j}$.

Following equations describe conditions for orthonormality. 
$\boldsymbol{V}_{0}^{T} \boldsymbol{K}_{0} \boldsymbol{V}_{0}=\boldsymbol{\Omega}_{0}^{2}$,

$\boldsymbol{V}_{0}^{T} \boldsymbol{M}_{0} \boldsymbol{V}_{0}=\boldsymbol{I}$,

$\boldsymbol{V}_{0}^{T} \boldsymbol{B}_{0} \boldsymbol{V}_{0}=2 \Delta=2\left(\alpha \boldsymbol{I}+\beta \Omega_{0}^{2}\right)$

Where $\Omega 00$ is the diagonal spectral matrix that includes natural frequencies $\boldsymbol{\omega}_{0} ;, \boldsymbol{V}_{\mathbf{0}}$ is the modal matrix including eigen modes $\boldsymbol{v}_{0 j}, \boldsymbol{B}_{0}$ is the damping matrix and $\Delta$ represents the matrix of constant decay elements $\boldsymbol{\delta}_{j}$, which are expressed by the following equation

$\delta_{j}=2 \xi \omega_{0 j}$

The symbol $\boldsymbol{\xi}$ introduce the comparative damping of the structure.

Because modifying substructures are not located evenly along the original structure, the resulting structure can be interpreted as a disproportionally damped system of $2 \mathrm{n}$ dimensional space represented by the coefficient matrices $N$ and $P$. The vibration of this system can be described by the following second order differential equation. where

$$
\boldsymbol{N} \dot{\boldsymbol{x}}-\boldsymbol{P x}=\boldsymbol{r}
$$

Where coefficient matrices are represented by $K$,

$$
\boldsymbol{P}=\left[\begin{array}{cc}
\boldsymbol{K} & \mathbf{0} \\
\mathbf{0} & \boldsymbol{M}
\end{array}\right], N=\left[\begin{array}{cc}
\boldsymbol{B} & \boldsymbol{M} \\
M & \mathbf{0}
\end{array}\right], \boldsymbol{x}=\left[\begin{array}{c}
\boldsymbol{q} \\
\dot{\boldsymbol{q}}
\end{array}\right], \boldsymbol{r}=\left[\begin{array}{c}
\boldsymbol{f} \\
\mathbf{0}
\end{array}\right]
$$

$M$ and $B$ and the variables $q, f$ express generalised displacement vector and the excitation force.

Eigenvalue problem solution for this system:

$$
\left(\boldsymbol{P}-s_{j} \boldsymbol{N}\right) \boldsymbol{w}_{j}=0, \quad\left(\boldsymbol{K}+s_{j} \boldsymbol{B}+\boldsymbol{s}_{j}^{2} \boldsymbol{M}\right) \boldsymbol{v}_{j}=\mathbf{0}
$$

Where $\boldsymbol{v}_{j}$ interpret jth eigen mode and imaginary part of its eigenvalue $\boldsymbol{s}_{j}$ express the angular frequency of damped system $\omega_{D j}$.

The following equations represents conditions for orthonormality.

$$
\begin{aligned}
& \boldsymbol{W}^{T} \boldsymbol{P} \boldsymbol{W}=\boldsymbol{S} \boldsymbol{W}^{T} \\
& \boldsymbol{W}^{T} \boldsymbol{N} \boldsymbol{W}=\boldsymbol{I} \\
& \boldsymbol{S} \boldsymbol{V}^{T} \boldsymbol{M} \boldsymbol{V}-\boldsymbol{V}^{T} \boldsymbol{K} \boldsymbol{V}=\boldsymbol{S} \\
& \boldsymbol{V}^{T} \boldsymbol{B} \boldsymbol{V}+\boldsymbol{V}^{T} \boldsymbol{M} \boldsymbol{V}+\boldsymbol{S} \boldsymbol{V}^{T} \boldsymbol{M} \boldsymbol{V}=\boldsymbol{I}
\end{aligned}
$$

Where $W$ and $V$ are modal matrices and $S$ represents the spectral matrix. These matrices can be expressed by relations:

$$
\boldsymbol{W}=\left\{\boldsymbol{w}_{j}\right\}=\left[\begin{array}{c}
\boldsymbol{V} \\
\boldsymbol{V} \boldsymbol{S}
\end{array}\right], \quad \boldsymbol{V}=\left\{\boldsymbol{v}_{j}\right\}, \quad \boldsymbol{S}=\operatorname{diag}\left(s_{j}\right)
$$

Relations (7) and (8) can be used to determine modal-spectral parameters of the resulting structure using original structure modal matrices $V_{0}$, $\Omega o, 2 \Delta$ and coefficient matrices of the modifying structure $\boldsymbol{M}_{N}, \boldsymbol{B}_{N}, \boldsymbol{K}_{N}$. Because dimensions of original structure matrices are usually different then coefficient matrices of the modifying structure, reduction methods as Guyan method or SEREP and zero elements expansions are required [1 - 3]. This process represents the modification of added substructure matrices $\boldsymbol{M}_{N}, \boldsymbol{B}_{N}, \boldsymbol{K}_{N}$ through $\boldsymbol{M}_{R}$, $\boldsymbol{B}_{R}, \boldsymbol{K}_{R}$ to $\boldsymbol{M}_{A}, \boldsymbol{B}_{A}, \boldsymbol{K}_{A}$, which can be graphically described as:

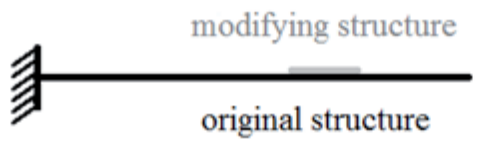

Fig. 1: Principal of modal synthesis.

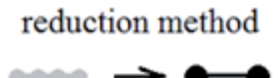

$\mathrm{Mv} \mathrm{BN} \mathrm{KN}_{\mathrm{N}} \rightarrow \mathrm{M}_{\mathrm{R}} \mathrm{B}_{\mathrm{R}} \mathrm{K}_{\mathrm{R}}$

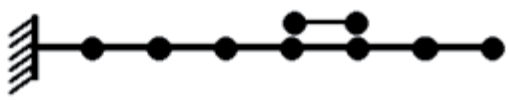

$\mathrm{M}_{\mathrm{R}} \mathrm{B}_{\mathrm{R}} \mathrm{K}_{\mathrm{R}} \longrightarrow \mathrm{M}_{\mathrm{A}} \mathrm{B}_{\mathrm{A}} \mathrm{K}_{\mathrm{A}}$

and express by equations:

$$
\boldsymbol{M}_{A}=\left[\begin{array}{cccc}
0 & 0 & 0 & 0 \\
0 & 0 & 0 & 0 \\
0 & 0 & \boldsymbol{M}_{R} & 0 \\
0 & 0 & 0 & 0
\end{array}\right], \boldsymbol{K}_{A}=\left[\begin{array}{cccc}
0 & 0 & 0 & 0 \\
0 & 0 & 0 & 0 \\
0 & 0 & \boldsymbol{K}_{R} & 0 \\
0 & 0 & 0 & 0
\end{array}\right], \boldsymbol{B}_{A}=\left[\begin{array}{cccc}
0 & 0 & 0 & 0 \\
0 & 0 & 0 & 0 \\
0 & 0 & \boldsymbol{B}_{R} & 0 \\
0 & 0 & 0 & 0
\end{array}\right]
$$


orthogonality conditions for disproportionally damped system:

$$
\begin{aligned}
& {\left[\boldsymbol{V}_{0}^{T} \boldsymbol{S} \boldsymbol{V}_{L}^{T}\right]\left[\begin{array}{cc}
-\left(\boldsymbol{K}_{P}+\boldsymbol{K}_{A}\right) & 0 \\
0 & \boldsymbol{M}_{P}+\boldsymbol{M}_{A}
\end{array}\right]\left[\begin{array}{c}
\boldsymbol{V} \\
\boldsymbol{V} \boldsymbol{S}
\end{array}\right]=\boldsymbol{S}} \\
& {\left[\boldsymbol{V}_{0}^{T} \boldsymbol{S} \boldsymbol{V}_{L}^{T}\right]\left[\begin{array}{cc}
-\left(\boldsymbol{B}_{P}+\boldsymbol{B}_{A}\right) & \boldsymbol{M}_{P}+\boldsymbol{M}_{A} \\
\boldsymbol{M}_{P}+\boldsymbol{M}_{A} & 0
\end{array}\right]\left[\begin{array}{c}
\boldsymbol{V} \\
\boldsymbol{V} \boldsymbol{S}
\end{array}\right]=\boldsymbol{I}}
\end{aligned}
$$

substitution using transformation matrix $\boldsymbol{T}_{L}$ :

$\boldsymbol{V}_{L}=\boldsymbol{V}_{0} \boldsymbol{T}_{L}$

condition for orthogonality then can be formed as:

$$
\begin{gathered}
{\left[\boldsymbol{T}_{L}^{T} \boldsymbol{S} \boldsymbol{T}_{L}^{T}\right]\left[\begin{array}{cc}
-\left(\Omega_{0 O}^{2}+\boldsymbol{V}_{0}^{T} \boldsymbol{K}_{A} \boldsymbol{V}_{0}\right) & 0 \\
0 & \boldsymbol{I}+\boldsymbol{V}_{0}^{T} \boldsymbol{M}_{A} \boldsymbol{V}_{0}
\end{array}\right]\left[\begin{array}{c}
\boldsymbol{T}_{L} \\
\boldsymbol{T}_{L} \boldsymbol{S}
\end{array}\right]=\boldsymbol{S}} \\
{\left[\boldsymbol{T}_{L}^{T} \boldsymbol{S} \boldsymbol{T}_{L}^{T}\right]\left[\begin{array}{cc}
\left.2 \Delta_{P}+\boldsymbol{V}_{0}^{T} \boldsymbol{B}_{A} \boldsymbol{V}_{0}\right) & \boldsymbol{I}+\boldsymbol{V}_{0}^{T} \boldsymbol{M}_{A} \boldsymbol{V}_{0} \\
\boldsymbol{I}+\boldsymbol{V}_{0}^{T} \boldsymbol{M}_{A} \boldsymbol{V}_{0} & 0
\end{array}\right]\left[\begin{array}{c}
\boldsymbol{T}_{L} \\
\boldsymbol{T}_{L} \boldsymbol{S}
\end{array}\right]=\boldsymbol{I}}
\end{gathered}
$$

Matrix $\boldsymbol{S}$ can be determined by the solution of following eigenvalue problem:

$$
\left(\boldsymbol{P}_{T}-s_{j} \boldsymbol{N}_{T}\right) \boldsymbol{w}_{T j}=0
$$

The transformation matrix and then the modal matrix can be figured out from equations (12) and (11).

In conclusion, the method of modal synthesis provides mode shapes and natural frequencies prediction of the resulting structure that consist of the original structure interpreted as a proportionally damped system and the modifying substructure described by its modal parameters obtained experimentally [3 -5$]$.

\section{Modal synthesis of the non-proportionately damped layered beam}

The above mentioned modal synthesis method can be used also in determining the modal and spectral properties of beam structures with added layers of vibroinsulation at specific points. A very simple illustration of such a system can be explained on an existing cantilever beam with a connected (added) beam which creates the modified beam. The presented method can be automated and used for the parameter optimization of vibroinsulating layers (orientation, geometry, material properties, etc...). The schematic representation of optimizing position $a$ and thickness $h$ of the vibroinsulating layer with respect to the maximum ratio of damping $\xi$ in the second Eigen mode is shown in Figure 2. (c).

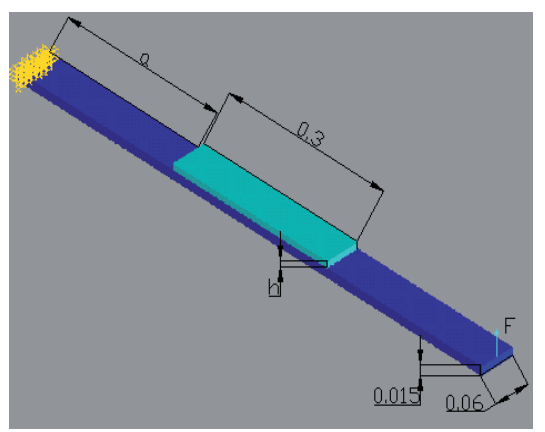

(a)

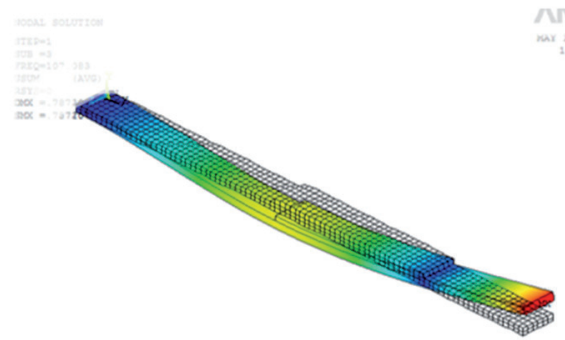

(b)

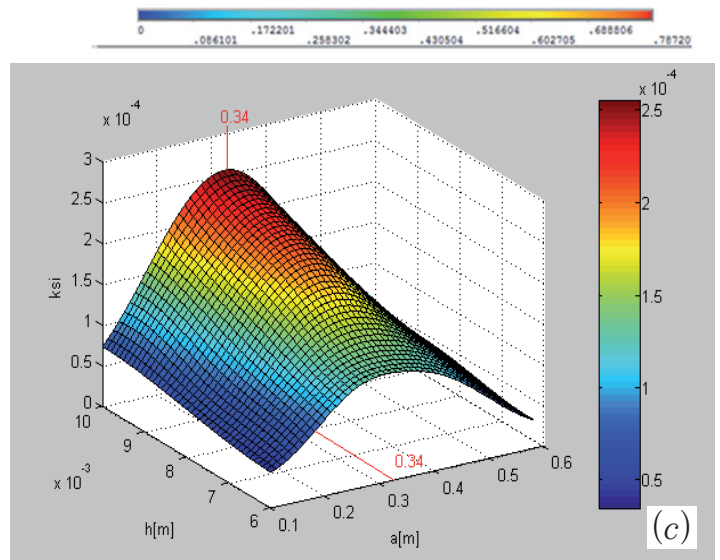

Fig. 2: Hierarchy $(a-c)$ for the optimization of position " $a$ " and thickness " $h$ " of the vibro-isolating layer.

It is obvious from dependence shown in Figure 2. (c) that by increasing the thickness of the isolating layer, the ratio of damping also gradually increases. More complex characteristics have values of proportional damping dependent on the location of the isolating layer, which directly depends on the corresponding eigen modes in the presented system. Figure 3. depicts the dependence of proportional damping of the 3rd and 4th modes 
and the position of the vibroinsulating layer. The steel beam with an aluminium foam layer was considered [6 - 8].

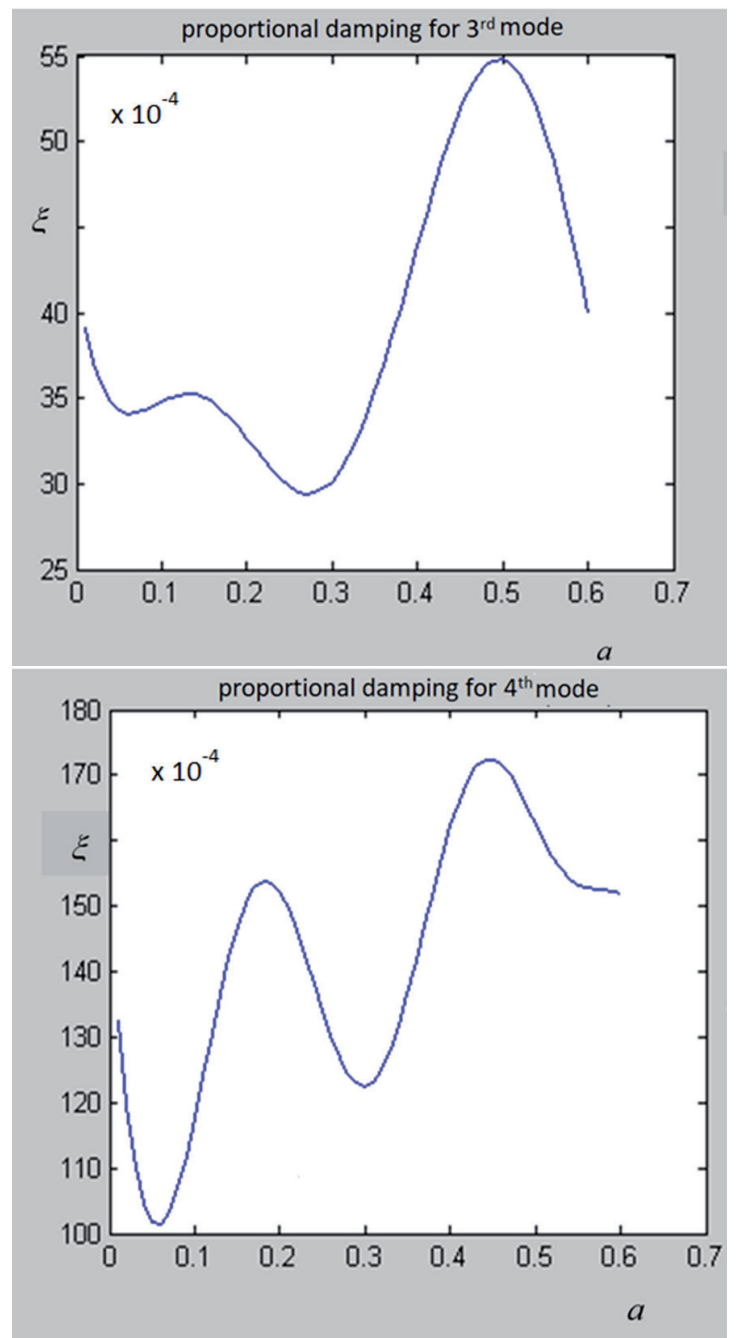

Fig. 3: Dependence of the proportional damping (3rd and 4th modes) on the position of the vibroinsulating layer.

From the presented properties, it can be seen how it is possible to achieve the desired damping of some Eigen modes by choosing the appropriate position and thickness of the vibroinsulating layer. Therefore, on the basis of modal synthesis it is possible, without any time-consuming calculations, to determine how effective the chosen parameters of the vibroinsulating layer are and in what manner do the dynamic properties of the modifies structure change.

\section{Numerical solution of modal-spectral parameters tuning of the modified structure}

Structural modification of the beam by aluminium foam layers causes the change of dynamic parameters of the original structure, which is known as well as structural dynamic modification. Modal analysis of the one-meter long cantilever beam was chosen to show dynamic parameters change using a structural dynamic modification, where first three modes of bending oscillation were analysed. Modifying structures, aluminium foam layers, were added to anti-node locations of the original structure to influent its stiffness matrix, which leads to increasing of natural frequencies (beam stiffening). This change of natural frequencies is used to avoid resonances and to decrease oscillation displacements. The first three mode shapes of the original beam and of its modifications with their natural frequencies are illustrated in the figure below (Fig. 4).

Structural modification of the beam by aluminium foam layers causes the change of dynamic parameters of the original structure, which is known as well as structural dynamic modification. Modal analysis of the one-meter long cantilever beam was chosen to show dynamic parameters change using a structural dynamic modification, where first three modes of bending oscillation were analysed. Modifying structures, aluminium foam layers, were added to anti-node locations of the original structure to influent its stiffness matrix, which leads to increasing of natural frequencies (beam stiffening). This change of natural frequencies is used to avoid resonances and to decrease oscillation displacements. The first three mode shapes of the original beam and of its modifications with their natural frequencies are illustrated in the figure below (Fig. 5).

The glue bond damage cause even the decreasing of natural frequencies, e.g. beam softening, which is an undesirable phenomenon.

\section{Conclusions}

The structural dynamic modification methodology presented in this work allows the desired dynamic parameters tuning of already made constructions by adding substructures. This method, which is based on modal synthesis, can be used in the case of combining the measured modal properties of the original system and final element model of 


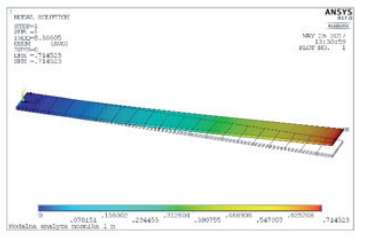

$8.3881 \mathrm{~Hz}$

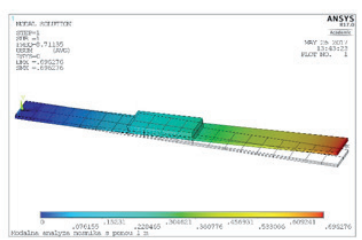

$8.7114 \mathrm{~Hz}$

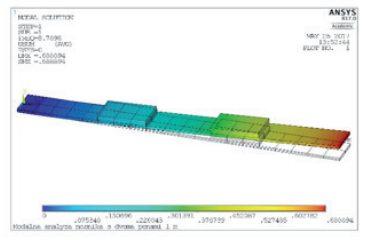

$8.7898 \mathrm{~Hz}$

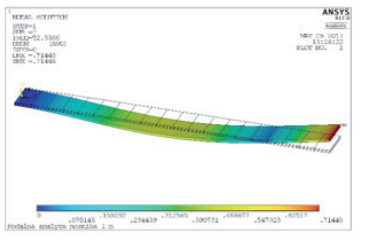

$52.539 \mathrm{~Hz}$

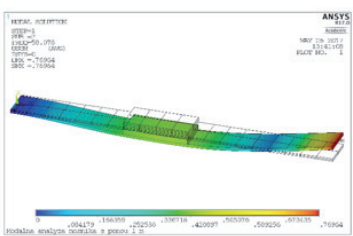

$58.078 \mathrm{~Hz}$

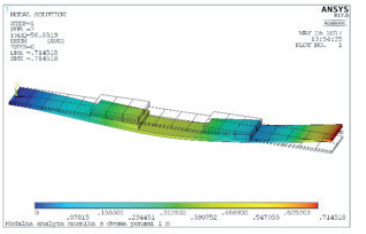

$56.832 \mathrm{~Hz}$

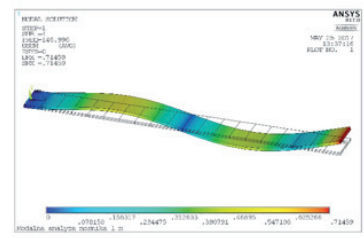

$147.00 \mathrm{~Hz}$

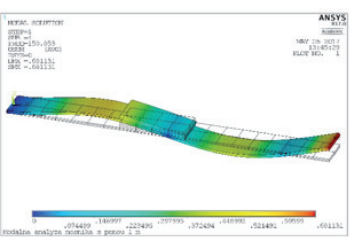

$150.85 \mathrm{~Hz}$

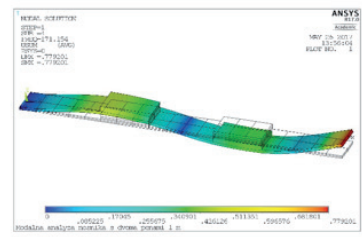

$171.15 \mathrm{~Hz}$

Fig. 4: Structural dynamic modification of the cantilever beam.

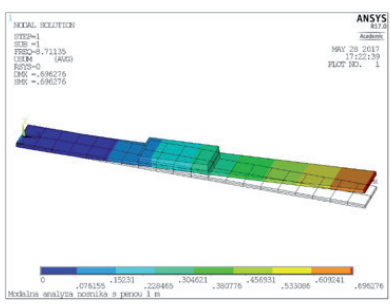

$8.329 \mathrm{~Hz}$

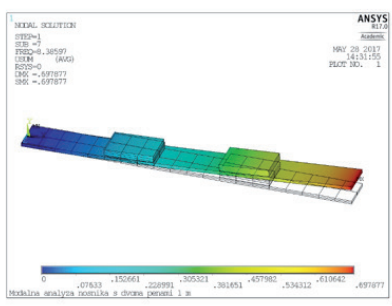

$8.386 \mathrm{~Hz}$

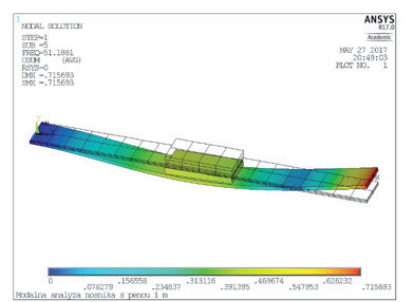

$51.021 \mathrm{~Hz}$

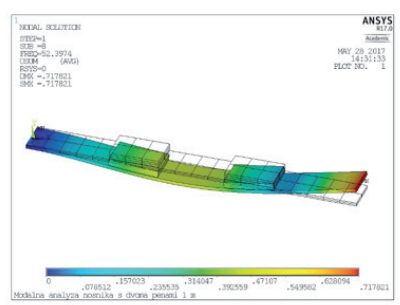

$52.397 \mathrm{~Hz}$

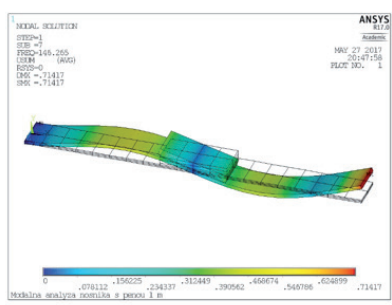

$146.19 \mathrm{~Hz}$

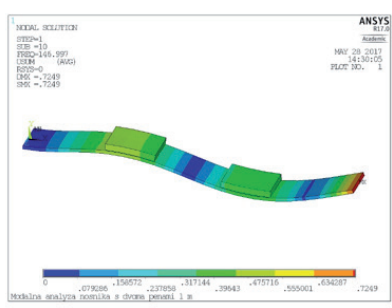

$146.89 \mathrm{~Hz}$

Fig. 5: Effect of the damaged glue layer.

the added substructure components. The work describes the application of the mentioned method to the situation of a cantilever beam modified with added aluminum foam layers. This paper also includes the final element model of the cantilever beam structure to express changes of modal parameters by adding aluminum substructures to the appropriate places to reach the stiffening of the beam. This article also examines the potential risk of using the structural modification by adding the substructures. There can occur a situation when glue bonds are damaged due to unsuitable conditions, 
which can lead to the reducing of the reinforcement effect or even to the softening of the original beam structure.

\section{Acknowledgments}

This work was supported by the grant from the Grant Agency of VEGA no. 1/0742/15 and by the Slovak Research and Development Agency under the contract no. APVV-15-0630.

\section{References and Notes}

[1] Curnier, A. On Three Modal Synthesis Variants. Journal of Sound and Vibration, 90, 1983

[2] Avitabile, P. Twenty Years of Structural Dynamic Modification - A Review. University of Massachusetts Lowell, Lowell, Massachusetts

[3] Slavík J., Stejskal V., Zeman V., Základy dynamiky strojú, ČVúT 1997

[4] Lancaster P.: Theory of Matrices. New York: Acad. Press, 1969

[5] Braun, S., Ewins, D., RAO, S. S.: Encyclopedia of Vibration. New York: Academic Press, 2002

[6] Kirch, U.: Structural Optimization. Berlin: Springer-Verlag, 1993

[7] Starek, L., Inman, D. J., Musil, M.: Updating of Non-Conservative Structure via Inverse Methods with Parameter Subset Selection. 5th International Conference on Sound and Vibration 3, USA, 1997

[8] Properties of Alulight foams, www.reade.com

[9] Zienkievicz, O. C.: The Finite Element Method. New York: McGraw-Hill, 1977

\section{Biographical notes}

Miloš Musil, prof., Ing., PhD., (born in 1962) has completed academic study at Slovak University of Technology, Faculty of Mechanical Engineering with honours (Ing.) in study programme Applied Mechanics (1986). Doctorate study was graduated in Applied Mechanics (1994) and he was granted with academic title Ph.D. From 2001 until now he is an Associate Professor at Institute of Applied Mechanics and Mechatronics at Faculty of Mechanical Engineering of the Slovak University of Technology in Bratislava. His work is dedicated to scope of dynamics of machines, their passive and active vibro-isolation, experimental identification of parameters and correction of mathematical models of mechanical and mechatronic system applied to evaluation of seismic resistance of structures, rotor dynamics, instability analysis of break squealing of vehicles or airplane wings, defect detection of mechanical systems or passive or active semi-active vehicle suspension. He is an author of various scientific papers (approx. 70) dedicated to mentioned scopes, which were published in national or international conferences. He is an author and co-author of 2 monographs (Active and semi-active vehicle suspension, Design of semi-active damper in vehicle suspension considering the tire lift off) and 3 textbooks. He was a responsible solutionist of various scientific projects in the previous term. Projects such as Safety increase of equipment in nuclear power plants during seismic activity, Influence of thermal loading on dynamics of automotive disc brakes and squeal noise generation, Failure detection of machine structures (cooperation with Virginia Polytechnic Institute and State University in USA) belong to his most remarkable projects.

René Havelka, Ing., (born in 1993) is a PhD student at the Slovak University of Technology, Faculty of Mechanical Engineering, Institute of Applied Mechanics and Mechatronics. He has graduated from the Faculty of Mechanical engineering in 2016. He is a specialist in the field of modal and spectral parameters tuning of structures. He uses also final element method to solve the research and practical issues. He is an author or co-author of 4 publications in journals and conferences.

Ondrej Chlebo, Ing., PhD., (born in 1986) is an assistant at the Slovak University of Technology, Faculty of Mechanical Engineering, Institute of Applied Mechanics and Mechatronics. He has graduated from the Faculty of Mechanical Engineering with the honour Ing. in 2009. His specialisation is the field of noise and vibration. His work is supported by the platform B\&K Pulse. The most significant project he participated on was Security Increasing of Nuclear Energy Devices during Seismic Event. He is the author of 20 publications about lowfrequency noise. He presented his results in several abroad conferences.

Ferdinand Havelka, Ing., PhD., (born in 1986) is an assistant at the Slovak University of Technology, Faculty of Mechanical Engineering, Institute of Applied Mechanics and Mechatronics. He has graduated from the Faculty of Mechanical Engineering with the honour Ing. in 2009. He is a specialist in the field of random vibration and mechatronics. He is an author of several articles published in local and abroad journals. He is also the author of 12 publications presented in local and abroad conferences.

Branislav Hučko, doc., Ing., PhD., (born in 1961) is a professor at the Slovak University of Technology, Faculty of Mechanical Engineering, Institute of Applied Mechanics and Mechatronics. He has graduated from the Faculty of Mechanical Engineering with honour Ing. in 1985. He is a specialist in the field of biomechanics and strength and elasticity supported by final element method. He has also participated on 4 textbooks, 4 utility models and 58 publications in journals and conferences in Slovakia or abroad. He has been guaranteed 7 research projects such as Development of retractor for operations un the abdominal cavity, Mechanical properties of foam composite materials and Micro electro-mechanic system (MEMS) of energy accumulation for medicine applications. 